Gut and Liver, Vol. 9, No. 4, July 2015, pp. 478-485

\title{
Efficacy of 7-Day and 14-Day Bismuth-Containing Quadruple Therapy and 7-Day and 14-Day Moxifloxacin-Based Triple Therapy as Second-Line Eradication for Helicobacter pylori Infection
}

Seong tae Lee, Dong Ho Lee, Ji Hyun Lim, Nayoung Kim, Young Soo Park, Cheol Min Shin, Hyun Jin Jo, and In sung Song Division of Gastroenterology, Department of Internal Medicine, Seoul National University Bundang Hospital, Seongnam, Korea

Background/Aims: Bismuth-containing quadruple and moxifloxacin-based triple regimens are recommended as second-line therapy for Helicobacter pylori infection. The aim of this study was to compare the efficacy of each regimen. Methods: From August 2004 to October 2012, a total of 949 patients (mean age, $54.32 \pm 12.08$ years; male, 49.4\%) who failed $H$. pylori eradication with a standard triple regimen were included. Patients treated with a bismuth-containing quadruple regimen for 7 and 14 days were designated as 7-BMT and 14-BMT, respectively, and those treated with a moxifloxacin-based triple regimen for 7 and 14 days were designated as 7-MA and 14-MA, respectively. H. pylori eradication was confirmed using the ${ }^{13} \mathrm{C}$-urea breath test, rapid urease test or histology. Results: The eradication rates by 7-BMT, 14-BMT, 7-MA, and 14-MA were 66.4\% (290/437), $71.1 \%$ (113/159), 53.1\% (51/96), and 73.5\% (189/257), respectively, by intention-to-treat analysis (ITT) and $76.5 \%$ (284/371), 83.8\% (109/130), 55.6\% (50/90), and 80.6\% (187/232), respectively, by per-protocol analysis (PP). The eradication rates were higher in 14-BMT than 7-BMT by the ITT and PP analyses ( $p=0.277$ and $p=0.082$, respectively). The 14-BMT and 14-MA treatments showed similar efficacies by ITT and PP ( $p=0.583$ and $p=0.443$, respectively). Conclusions: The 7-BMT, 14-BMT, and 14-MA treatments showed similar and suboptimal efficacies. In both regimens, extending the duration of treatment may be reasonable considering the high level of antibiotic resistance in Korea. (Gut Liver 2015;9:478-485)

Key Words: Helicobacter pylori; Second-line therapy; Bismuth-containing quadruple regimen; Moxifloxacin-based triple regimen

\section{INTRODUCTION}

Helicobacter pylori has been known to be associated with gastritis, peptic ulcer disease (gastric ulcer, duodenal ulcer), gastric mucosa-associated lymphoid tissue lymphoma, and gastric adenocarcinoma. ${ }^{1}$ Current guidelines recommend eradication therapy not only in these conditions but also in first-degree relatives of patients with gastric cancer. Furthermore, eradication of $H$. pylori should be considered in the patients with long-term therapy of nonsteroidal anti-inflammatory drugs (NSAIDs) or acetylsalicylic acid for reducing peptic ulcer and upper gastrointestinal bleeding. ${ }^{2-4}$

In Korea, the standard first-line therapy consisting of proton pump inhibitor (PPI), amoxicillin and clarithromycin has been generally used for 7 days. ${ }^{2,3,5,6}$ However, during the last few years, this PPI-based triple therapy regimen failed to achieve eradication rate $\geq 80 \%$ in several large clinical trials and metaanalyses. ${ }^{7,8}$ Moreover, in the clinical setting the failure rate of standard first-line therapy can in fact be higher and several studies have reported the decreasing efficacy of this regimen to lower than 75\% and even to lower than $50 \%$ on an intentionto-treat (ITT) basis. ${ }^{9-12}$ These unsatisfactory results are increasing the needs for second-line treatment options.

At present, the internationally recommended salvage eradication therapy for $H$. pylori is bismuth-containing quadruple regimen consisting of PPI, bismuth citrate, tetracycline and metronidazole for 7 to 14 days. ${ }^{2,13}$ Although this treatment regimen has been shown to be effective, a pooled analysis of 40 trials reported variable eradication rates and an average eradication rate of 76\% for bismuth-containing quadruple therapy as secondline treatment. ${ }^{14,15}$ At this point in time, newer therapeutic regimens using fluoroquinolones were suggested for eradication of H. pylori. Moxifloxacin (Bayer HealthCare AG, Wuppertal, Ger-

Correspondence to: Dong Ho Lee

Department of Internal Medicine, Seoul National University Bundang Hospital, 82 Gumi-ro 173beon-gil, Bundang-gu, Seongnam 463-707, Korea

Tel: +82-31-787-7006, Fax: +82-31-787-4051, E-mail: dhljohn@yahoo.co.kr

Received on January 9, 2014. Revised on February 22, 2014. Accepted on March 11, 2014. Published online July 25, 2014

pISSN 1976-2283 eISSN 2005-1212 http://dx.doi.org/10.5009/gnl14020

() This is an Open Access article distributed under the terms of the Creative Commons Attribution Non-Commercial License (http://creativecommons.org/licenses/by-nc/4.0) which permits unrestricted non-commercial use, distribution, and reproduction in any medium, provided the original work is properly cited. 
many) is a second-generation fluoroquinolone launched in 1999 and widely used to treat infections of the respiratory tract and skin. ${ }^{16,17}$ It penetrates tissues well and its long half-life, 9 to 16 hours allows once-daily dosing. ${ }^{18-20}$ Recently, some reports have shown that moxifloxacin-based triple regimen has good efficacy for the treatment of $H$. pylori infection as second-line treatment. ${ }^{20-22}$ A meta-analysis showed an advantage of levofloxacin and moxifloxacin-based triple therapy for eradication of $H$. pylori as second-line treatment; but, this meta-analysis included only three trials, and did not analyze moxifloxacin-based triple therapy separately. ${ }^{23}$ Data about moxifloxacin-based triple therapy as second-line treatment are still lacking. Few recent studies suggested an advantage of moxifloxacin-based triple therapy compared with bismuth-containing quadruple therapy in terms of efficacy, side effects and compliance. ${ }^{21,22,24}$

However, resistance to fluoroquinolones among $H$. pyloriinfected patients has been increasingly reported in countries where it is used frequently and limiting its application. ${ }^{25-28}$

It is known that the Korean population has a high risk for $H$. pylori infection and a high prevalence of antibiotic resistance. ${ }^{29,30}$ In this regard, it may be reasonable to investigate whether the eradication rate has fallen with second-line eradication therapy and extending the duration of therapy can increase the eradication rate.

The aim of this study was to compare the efficacy, compliance and adverse events of bismuth-containing quadruple therapy with moxifloxacin-based triple therapy according to the duration of treatment as second-line treatment for a Korean study population of the recent 9 years.

\section{MATERIALS AND METHODS}

\section{Study population}

This was a retrospective study in which consecutive patients who fulfilled the following inclusion criteria were recruited using a computer-generated table during the period from August 2004 to October 2012 at Seoul National University Bundang Hospital in Korea. We reviewed all the patients who were at least 18 years of age and initially failed to eradicate $H$. pylori with standard triple therapy (PPI twice a day as usual dose, amoxicillin $1 \mathrm{~g}$ twice a day and clarithromycin $500 \mathrm{mg}$ twice a day). Usual doses of rabeprazole, esomeprazole, pantoprazole, lansoprazole and omeprazole were used. Of these patients, a total of 949 patients who were treated with second-line eradication therapy using bismuth-containing quadruple regimen or moxifloxacin-based triple regimen for 7 or 14 days were enrolled.

Failure to eradicate $H$. pylori was defined according to one or more of the following three tests: (1) a positive rapid urease test (CLO test; Delta West, Bentley, Australia) by gastric mucosal biopsy from the antrum or body; (2) a positive ${ }^{13} \mathrm{C}$-urea breath test; or (3) a positive histological evidence of $H$. pylori by modi- fied Giemsa staining in the antrum or body.

The exclusion criteria were (1) the use of $\mathrm{H}_{2}$-receptor antagonists, PPIs, NSAIDs, or antibiotics during the previous 4 weeks; (2) advanced gastric cancer or previous gastric surgery; (3) the presence of systemic illness such as liver cirrhosis or chronic renal failure; (4) pregnant or breast-feeding women; (5) age $<18$ years; or (6) any condition possibly correlated with poor compliance such as alcoholics or drug abusers.

\section{Treatment and follow-up}

Four groups of patients treated with second-line eradication therapy were named using acronyms. Bismuth-containing quadruple therapy consisting of tripotassium dicitrato bismuthate (DENOL ${ }^{\circledR}$; Greencross Co., Seoul, Korea) $300 \mathrm{mg} 4$ times a day (three tabs 30 minutes before meals and one tab 2 hours after dinner), tetracycline $500 \mathrm{mg} 4$ times a day, metronidazole $500 \mathrm{mg} 3$ times a day and usual dose of PPI twice a day for 7 days was named as 7-BMT and that for 14 days as 14-BMT, respectively. Moxifloxacin-based triple therapy consisting of moxifloxacin $400 \mathrm{mg}$ every day, amoxicillin 1,000 mg twice a day and usual dose of PPI twice a day for 7 days was named as 7-MA and that for 14 days as 14-MA, respectively.

The 949 patients were assigned to one of the following four treatment regimens as second-line eradication: 7-BMT $(\mathrm{n}=437)$; 14-BMT ( $\mathrm{n}=159)$; 7-MA ( $\mathrm{n}=96)$; and 14-MA ( $\mathrm{n}=257)$. H. pylori eradication was defined as a negative ${ }^{13} \mathrm{C}$-urea breath test or negative rapid urease test or negative histology at least 4 weeks after completion of therapy.

Additionally, compliance with therapy was assessed by direct questions by a physician and pill count 1 week or 2 weeks after completion of therapy. Good compliance was considered when patient intake the drug more than $85 \%$. At the same time, adverse events of the patients were reviewed.

\section{3. ${ }^{13} \mathrm{C}$-urea breath test}

No PPI, bismuth, $\mathrm{H}_{2}$-blocker, and antibiotics were allowed within 4 weeks before the urea breath test and before the test patient was fasted for 4 hours. Test meal was not given, and a predose breath sample was obtained. Then patient administered $75 \mathrm{mg}$ of ${ }^{13} \mathrm{C}$-urea powder (Helikit ${ }^{\mathrm{TM}}$; Isotechnika, Edmonton, Canada) dissolved in $50 \mathrm{~mL}$ of water, orally. Thirty minutes later, a second breath sample was collected. The cutoff value used was $4 \% 0^{31}$ The collected samples were analyzed by means of an isotope ratio mass spectrometer (Heliview ${ }^{\circledR}$; Medichems, Seoul, Korea).

\section{Urease test}

Two biopsy specimens, one each from the antrum and the body, were used for the rapid urease test (CLO test). Antral and body biopsy specimens were evaluated separately, and color change of all urease tests were monitored for up to 24 hours. 


\section{Histological evaluation}

Two biopsy specimens obtained from the antrum and the body were fixed in formalin and submitted to two experienced pathologists for histological examination. They assessed the presence of $H$. pylori using modified Giemsa staining and the degree of inflammatory cell infiltration, atrophy and intestinal metaplasia with hematoxylin and eosin stain.

\section{Statistical analysis}

The analysis was conducted using SPSS version 18.0 for Windows (SPSS Inc., Chicago, IL, USA). $H$. pylori eradication rate was analyzed on an ITT basis and on a per-protocol (PP) basis. The ITT analysis included all patients enrolled in the study, and patients with an unproven state of $H$. pylori eradication due to follow-up loss were considered as treatment failure for the ITT analysis. The PP analysis excluded patients who were unavailable data of $H$. pylori eradication state because of follow-up loss, took less than $85 \%$ of treatment drugs and discontinued therapy. Baseline characteristics were compared using chisquare, Fisher exact, and ANOVA test. Outcomes, adverse events and drug compliance among 7-BMT, 14-BMT, 7-MA, and 14MA were analyzed by chi-square and Fisher exact test. Additionally, linear-by-linear association was used to evaluate the linear trend of eradication rates by year. A p-value of less than 0.05 was considered statistically significant.

\section{RESULTS}

\section{Patients}

Finally, 949 eligible patients were reviewed in this study group from August 2004 to October 2012. The schematic diagram of the study population is shown in Fig. 1, and baseline characteristics of the study population are summarized in Table 1. Baseline characteristics of age, comorbidities (hypertension, diabetes mellitus), and alcohol intake were similar, but male gender, current smoking, and endoscopic diagnosis were different among the four treatment groups; male gender (49.2\% vs $61.6 \%$ vs $50.0 \%$ vs $42.0 \%, \mathrm{p}=0.002$ ), current smoking ( $12.4 \%$ vs $13.8 \%$ vs $8.3 \%$ vs $5.4 \%, p=0.011)$ and endoscopic diagnosis $(\mathrm{p}<0.001)$.

\section{H. pylori eradication rates}

H. pylori eradication rates are listed in Table 2, and Fig. 2 shows eradication rates of $H$. pylori according to each therapeutic regimen.

The ITT eradication rates were $66.4 \%$ (95\% confidence interval [CI], 61.8 to $70.5 ; 290 / 437)$ and $71.1 \%$ (95\% CI, 63.5 to 77.4; 113/159) between 7-BMT and 14-BMT ( $p=0.277$ ), 53.1\%

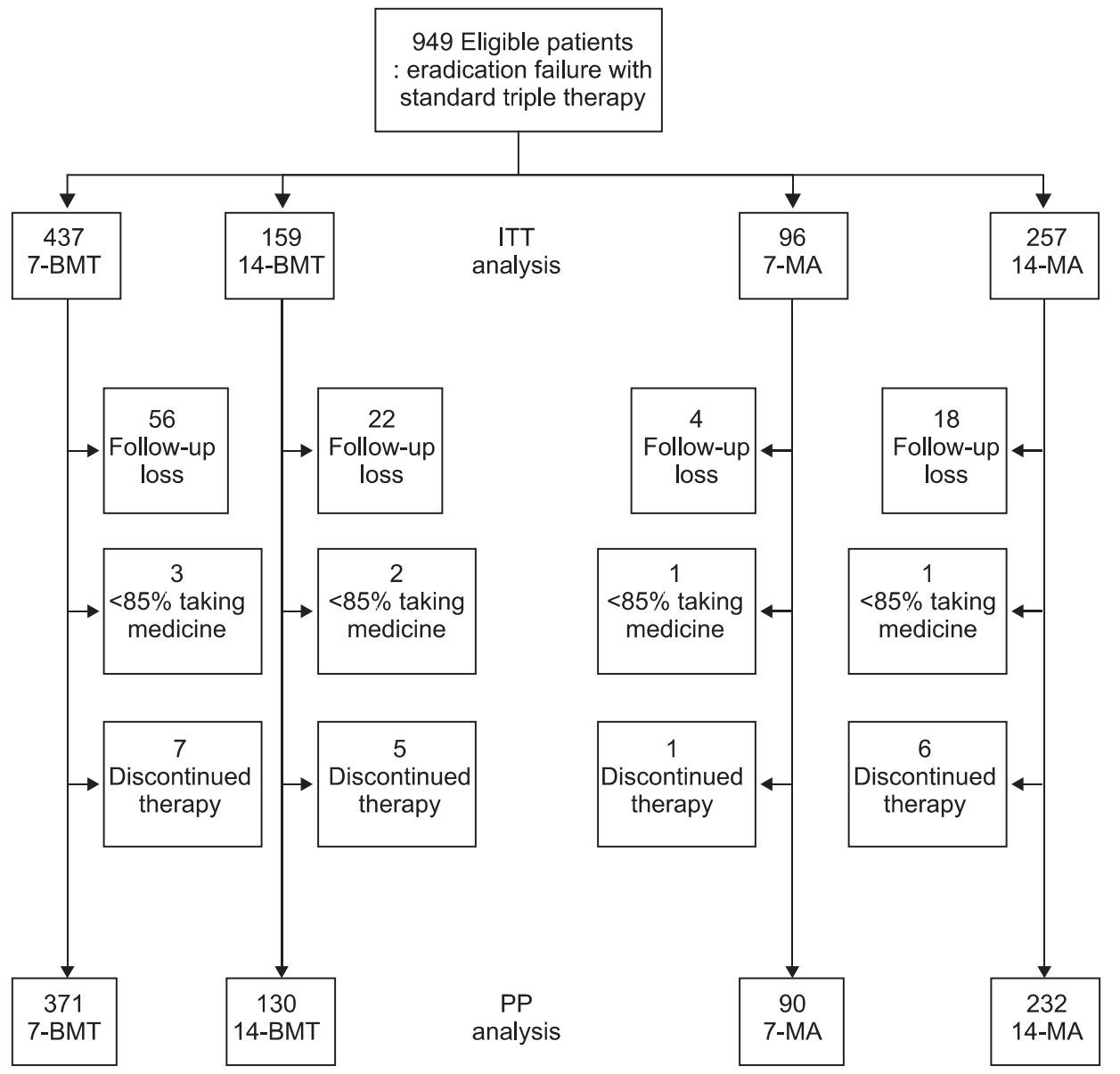

Fig. 1. Schematic diagram of the study population.

7-BMT, 7-day bismuth-containing quadruple therapy; 14-BMT, 14day bismuth-containing quadruple therapy; 7-MA, 7-day moxifloxacinbased triple therapy; 14-MA, 14-day moxifloxacin-based triple therapy; ITT, intention-to-treat; PP, perprotocol. 
Table 1. Baseline Characteristics of the Study Population

\begin{tabular}{|c|c|c|c|c|}
\hline Characteristic & 7-BMT & 14-BMT & 7-MA & 14-MA \\
\hline No. of patients & 437 & 159 & 96 & 257 \\
\hline Age, yr & $54.0 \pm 11.7$ & $53.7 \pm 12.2$ & $54.2 \pm 12.2$ & $55.4 \pm 12.5$ \\
\hline Gender, male/female & $215 / 222$ & $98 / 61$ & $48 / 48$ & $108 / 149$ \\
\hline \multicolumn{5}{|l|}{ Comorbidity } \\
\hline Hypertension & 94 (21.5) & $33(20.8)$ & $13(13.5)$ & $58(22.6)$ \\
\hline Diabetes & $37(8.5)$ & $21(13.2)$ & $6(6.3)$ & $20(7.8)$ \\
\hline Current smoking & $54(12.4)$ & $22(13.8)$ & $8(8.3)$ & $14(5.4)$ \\
\hline Alcohol intake & $98(22.4)$ & $34(21.4)$ & 19 (19.8) & 39 (15.2) \\
\hline \multicolumn{5}{|l|}{ Endoscopic diagnosis } \\
\hline HPAG & $196(44.9)$ & 79 (49.7) & $62(64.6)$ & 153 (59.5) \\
\hline Gastric ulcer & 68 (15.6) & $10(6.3)$ & $12(12.5)$ & 29 (11.3) \\
\hline Duodenal ulcer & $147(33.6)$ & $52(32.7)$ & 19 (19.8) & 53 (20.6) \\
\hline $\mathrm{GU}+\mathrm{DU}$ & $17(3.9)$ & $8(5.0)$ & $2(2.1)$ & $7(2.7)$ \\
\hline Early gastric cancer & $6(1.4)$ & $5(3.1)$ & $1(1.0)$ & $14(5.4)$ \\
\hline MALToma & $3(0.7)$ & $5(3.1)$ & 0 & 0 \\
\hline Carcinoid tumor & 0 & 0 & 0 & $1(0.4)$ \\
\hline
\end{tabular}

Data are presented as mean \pm SD or number $(\%)$.

7-BMT, 7-day bismuth-containing quadruple therapy; 14-BMT, 14-day bismuth-containing quadruple therapy; 7-MA, 7-day moxifloxacin-based triple therapy; 14-MA, 14-day moxifloxacin-based triple therapy; HPAG, Helicobacter pylori-associated gastritis; GU, gastric ulcer; DU, duodenal ulcer; MALToma, mucosa-associated lymphoid tissue lymphoma.

Table 2. Outcomes in the 7- and 14-Day Bismuth-Containing Quadruple and Moxifloxacin-Based Triple Therapies

\begin{tabular}{|c|c|c|c|c|c|}
\hline & 7-BMT & 14-BMT & 7-MA & 14-MA & $\mathrm{p}$-value \\
\hline \multicolumn{6}{|l|}{ Eradication rate } \\
\hline ITT analysis & 290/437 (66.4) & $113 / 159(71.1)$ & $51 / 96(53.1)$ & $189 / 257$ (73.5) & 0.002 \\
\hline $95 \%$ CI, \% & $61.8-70.5$ & $63.5-77.4$ & $42.7-63.5$ & $68.5-79.0$ & \\
\hline PP analysis & 284/371 (76.5) & 109/130 (83.8) & $50 / 90(55.6)$ & 187/232 (80.6) & $<0.001$ \\
\hline $95 \%$ CI, \% & 72.1-80.9 & $77.1-90.1$ & $45.5-65.5$ & $75.5-85.6$ & \\
\hline Compliance & $371 / 437$ (84.9) & 130/159 (81.8) & 90/96 (93.8) & 232/257 (90.3) & 0.009 \\
\hline Adverse events & & & & & $<0.001$ \\
\hline Nausea/vomiting & $12(2.7)$ & $10(6.3)$ & $2(2.1)$ & $19(7.4)$ & \\
\hline Diarrhea & $6(1.4)$ & $4(2.5)$ & $2(2.1)$ & $31(12.1)$ & \\
\hline Taste disturbance & $3(0.7)$ & $1(0.6)$ & 0 & $11(4.3)$ & \\
\hline Epigastric soreness & $12(2.7)$ & $6(3.8)$ & $1(1.0)$ & $17(6.6)$ & \\
\hline Bloating & 0 & $1(0.6)$ & 0 & $3(1.2)$ & \\
\hline Headache & 0 & $1(0.6)$ & $1(1.0)$ & $1(0.4)$ & \\
\hline Urticaria & $3(0.7)$ & $3(1.9)$ & 0 & $2(0.8)$ & \\
\hline Dizziness/weakness & $6(1.4)$ & $3(1.9)$ & $1(1.0)$ & $3(1.2)$ & \\
\hline Total & $42(9.6)$ & $29(18.2)$ & $7(7.3)$ & 87 (33.9) & \\
\hline
\end{tabular}

Data are presented as number $(\%)$.

7-BMT, 7-day bismuth-containing quadruple therapy; 14-BMT, 14-day bismuth-containing quadruple therapy; 7-MA, 7-day moxifloxacin-based triple therapy; 14-MA, 14-day moxifloxacin-based triple therapy; ITT, intention-to-treat; CI, confidence interval; PP, per-protocol.

(95\% CI, 42.7 to $63.5 ; 51 / 96)$ and $73.5 \%$ (95\% CI, 68.5 to 79.0 ; 189/257) between 7-MA and 14-MA ( $<<0.001)$, respectively. Additionally, the ITT eradication rates were $66.4 \%$ and $53.1 \%$ between 7-BMT and 7-MA ( $\mathrm{p}=0.014), 66.4 \%$ and $73.5 \%$ between 7-BMT and 14-MA ( $\mathrm{p}=0.048$ ), 71.1\% and 73.5\% between 14-BMT and 14-MA ( $\mathrm{p}=0.583)$. 
A

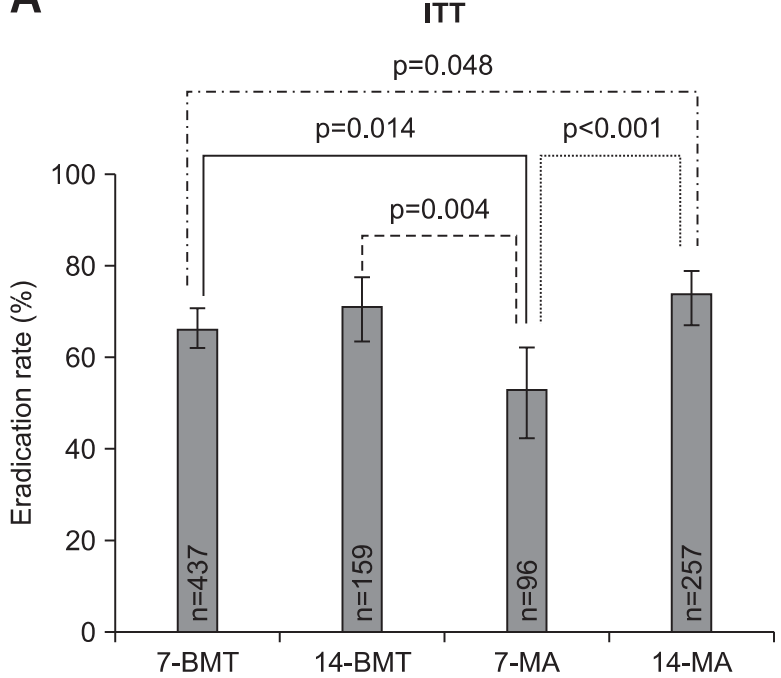

B

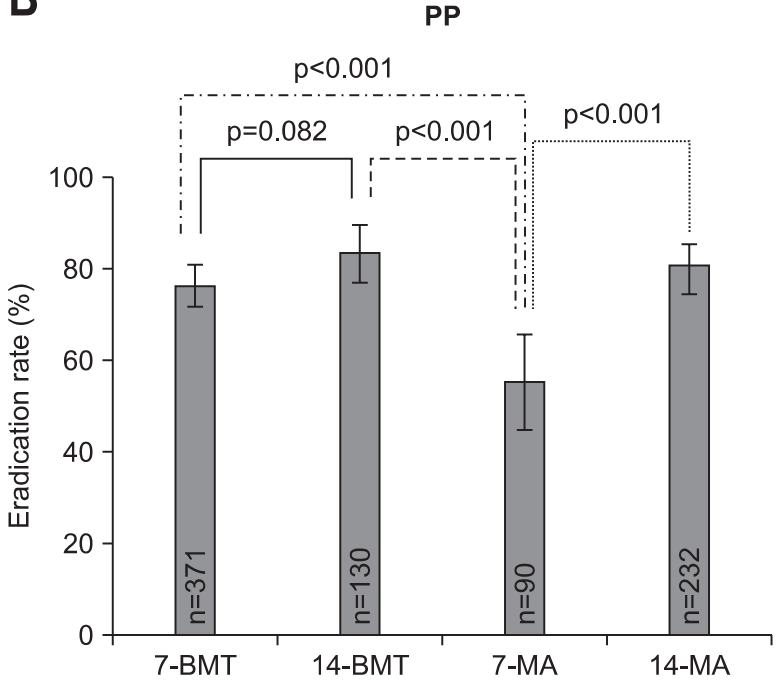

Fig. 2. Helicobacter pylori eradication rates according to the therapeutic regimen. (A) Intention-to-treat (ITT). (B) Per-protocol (PP). Error bars indicate the 95\% confidence interval.

7-BMT, 7-day bismuth-containing quadruple therapy; 14-BMT, 14-day bismuth-containing quadruple therapy; 7-MA, 7-day moxifloxacin-based triple therapy; 14-MA, 14-day moxifloxacin-based triple therapy.

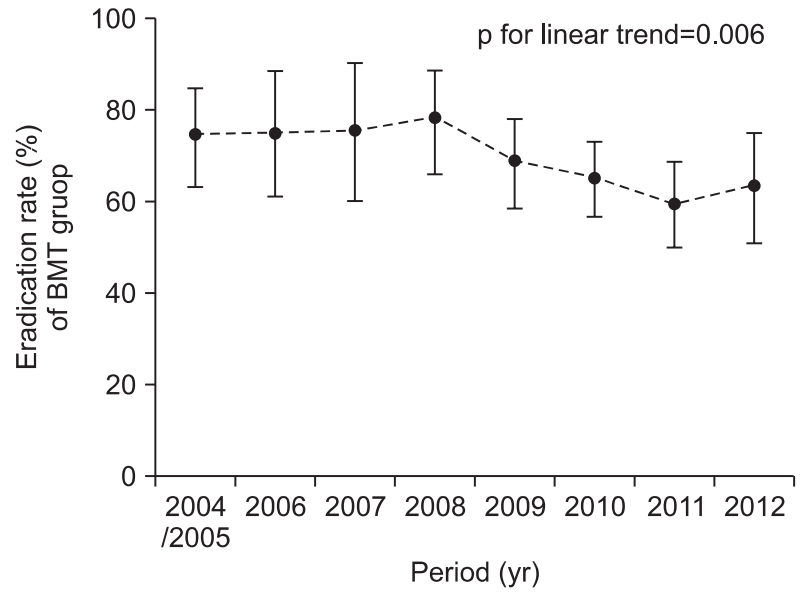

Fig. 3. The overall eradication rate of bismuth-containing quadruple therapy over time. The error bars indicate the 95\% confidence interval; the BMT group represents 7-day bismuth-containing quadruple therapy plus 14-day bismuth-containing quadruple therapy.

The PP eradication rates were $76.5 \%(95 \% \mathrm{CI}, 72.1$ to 80.9 ; 284/371) and 83.8\% (95\% CI, 77.1 to 90.1; 109/130) between 7-BMT and 14-BMT ( $\mathrm{p}=0.082$ ), 55.6\% (95\% CI, 45.5 to 65.5 ; $50 / 90)$ and $80.6 \%$ (95\% CI, 75.5 to $85.6 ; 187 / 232$ ) between 7-MA and 14-MA ( $<<0.001)$, respectively. Additionally, the PP eradication rates were $76.5 \%$ and $55.6 \%$ between 7-BMT and 7-MA ( $\mathrm{p}<0.001$ ), 76.5\% and 80.6\% between 7-BMT and 14MA ( $\mathrm{p}=0.242), 83.8 \%$ and $80.6 \%$ between 14-BMT and 14-MA $(\mathrm{p}=0.443)$.

Fig. 3 shows that the overall eradication rate of bismuth-containing quadruple therapy has been decreasing as time elapsed ( $p$ for linear trend=0.006). However, moxifloxacin-based triple therapy showed no statistical significance in the changes of eradication rate according to time ( $\mathrm{p}$ for linear trend=0.225).

\section{Compliance and adverse events}

The compliance of the BMT group (represents 7-BMT plus 14BMT) was inferior compared to that of MA group (represents 7-MA plus 14-MA); $84.1 \%$ vs $91.2 \%, p=0.002$. However, no significant differences were reported according to the duration of therapy within same therapeutic regimen.

The adverse events were recorded in 42 of 7-BMT (9.6\%) and 29 of 14-BMT (18.2\%) ( $\mathrm{p}=0.011)$ and in 7 of 7-MA (7.3\%) and 87 of 14-MA (33.9\%) ( $\mathrm{p}<0.001)$, respectively. Details about adverse events that occurred during the period of study are listed in Table 2. Generally, adverse events were reported in 71 of the bismuth-containing quadruple therapy group (11.9\%) and 94 of the moxifloxacin-based triple therapy group (26.6\%) $(\mathrm{p}<0.001)$.

The number of patients who discontinued therapy due to adverse events were 7 of 7-BMT (1.6\%), 5 of 14-BMT (3.1\%) $(\mathrm{p}=0.236), 1$ of $7-\mathrm{MA}(1.0 \%)$ and 6 of 14-MA (2.3\%) ( $\mathrm{p}=0.438)$, respectively. Generally, the number of patients who discontinued therapy due to adverse events were 12 in the bismuthcontaining quadruple therapy group (2.0\%) and 7 in the moxifloxacin-based triple therapy group (2.0\%) ( $p=0.974)$.

The prevalence of adverse events increased as the duration of therapy increased. The most frequent adverse events were nausea/vomiting in the 7-BMT and 14-BMT, while diarrhea was more frequent than nausea/vomiting in the 7-MA and 14-MA.

\section{DISCUSSION}

It is uncertain what is the best second-line therapy for $H$. pylori eradication after initial treatment failure with standard 
triple therapy containing PPI, amoxicillin, and clarithromycin. As mentioned earlier, bismuth-containing quadruple regimen has been commonly used as second-line therapy after the initial failure of $H$. pylori eradication ${ }^{2,13}$ and moxifloxacin-based triple regimen has been suggested as an alternative second-line therapeutic regimen. ${ }^{21-23}$ Some studies have reported about the efficacy of second-line eradication therapy between two groups; however, to our knowledge, this study is the first report comparing four groups simultaneously. In our study, 7-MA failed to show an appropriate eradication rate. The 14-BMT showed higher eradication rate than 7-BMT, even though this result was not statistically significant. The eradication rate of 14-MA was superior to that of 7-MA, and this result was statistically significant. In addition, 14-BMT and 14-MA showed similar efficacy by ITT and PP analysis.

These results can be explained by antibiotic resistance. Pretreatment resistance to antibiotics may be the most important factor in patients who do not respond to eradication therapy. ${ }^{32}$ This indicates that the choice of regimen for second-line eradication depends on what kind of regimen was used initially, as retreatment with the same regimen cannot be recommended. ${ }^{33}$ Resistance to antibiotics has been increasing in many countries as a major cause of eradication failure, ${ }^{7,34}$ but we have not investigated antibiotic resistance due to the retrospective nature of this study. Thus, this study referred to previous reports about antibiotic susceptibility of H.pylori in our hospital. ${ }^{30,35}$ A recent study at our center involving 269 patients showed a resistant rate of amoxicillin, metronidazole, and tetracycline was 13.8\%, 27.1\%, and 9.7\%, respectively. ${ }^{30}$ Another data in our center with 86 patients from 2003 to 2012 showed secondary resistance to antibiotics after failure of eradication. In this study, the secondary resistance rate to amoxicillin $(\mathrm{p}=0.175)$, moxifloxacin ( $\mathrm{p}=0.025)$, and metronidazole $(\mathrm{p}=0.034)$ have all been observed to increase over time. ${ }^{35}$ It is known that bacterial resistance to metronidazole has a negative impact on the effectiveness of bismuth-containing quadruple therapy, but it is possible to overcome this drawback by increasing the duration of treatment. $^{36}$ The resistance rate of metronidazole is $20 \%$ to $40 \%$ in the United States and Europe, but the prevalence is higher from $50 \%$ to $80 \%$ in developing countries. ${ }^{37}$ Therefore, it may be expected that the increased duration of bismuth-containing quadruple therapy would be more effective than shorter treatment duration in high metronidazole resistant areas, and for this reason the variable results for eradication rate according to treatment duration might be occurred. With regard to tetracycline resistance, it is suggested that tetracycline does not have a major role in $H$. pylori eradication. ${ }^{38}$ However, further studies are needed to clarify the impact of tetracycline on $H$. pylori eradication.

Similarly, resistance of $H$. pylori to fluoroquinolones was reported to vary depending on different countries or regions. ${ }^{39}$ In previous studies, moxifloxacin-based triple therapy as first-line treatment for $H$. pylori infection showed eradication rates of about 90\%. ${ }^{18,19}$ Accordingly, moxifloxacin or levofloxacin-based triple therapy was recommended as an alternative second-line therapeutic regimen after failure of primary eradication therapy. However, some studies have reported that these triple therapies are not superior to previous established therapies. ${ }^{40}$ These results might be associated with increasing resistance to fluoroquinolones, especially in Korea. The prevalence of fluoroquinoloneresistance is high in Asia except China. ${ }^{41-43}$ These regional difference could be related to the proportion of patients taking these drugs since fluoroquinolone-resistance can be easily acquired. ${ }^{44}$ A previous study reported that 10-day moxifloxacin-based triple therapy showed $71.9 \%$ of eradication rate by ITT analysis in our hospital. ${ }^{24}$ Furthermore, in vitro study regarding susceptibility to fluoroquinolones performed in our hospital revealed an increasing resistant rate over time and getting up to 50\% secondary resistant rate of $H$. pylori against moxifloxacin. ${ }^{35}$ For these reasons, this study may not show satisfactory eradication rates of moxifloxacin-based triple therapy.

In addition, poor compliance is also another important factor in treatment failure. ${ }^{36,45}$ Moxifloxacin-based triple therapy is more simpler than bismuth-containing quadruple therapy in the method of taking medicine, while bismuth-containing quadruple therapy entails a complex scheme in relation to the number of tablets and the method of administration, causing poor patient compliance. ${ }^{36,45}$ This study also showed that the MA group had better compliance than the BMT group, and revealed no significant difference according to treatment duration within the same regimen.

In our study, we found a higher rate of adverse events with 14 days compared to 7 days of treatment duration in each therapeutic regimen. Adverse events were increased as the duration of therapy was prolonged, but the rates of discontinued medication according to the duration of therapy were not significantly different. These phenomena may be due to the fact that most side effects causing discontinue eradication therapy appear in the early phase of treatment.

The limitation of our study is its retrospective design. There were 77/596 (12.9\%) follow-up losses in the BMT group and $22 / 353(6.2 \%)$ in the MA group ( $p<0.001)$. The overall adverse events were 71/596 (11.9\%) in the BMT group compared with $94 / 353(26.6 \%)$ in the MA group $(\mathrm{p}<0.001)$. Adverse events could be lower in BMT group because the patients who experienced adverse events might be exist in follow-up loss patients. Furthermore, limitation of the retrospective nature of this study hindered collecting interviews for adverse events. The adverse events may also be undervalued because no record about adverse events was regarded as none of them. For these reasons, 14-MA may show more adverse events than 14-BMT, which is a somewhat different finding from other studies. ${ }^{21,22,24}$ Therefore, assessment regarding adverse events through our study cannot be considered appropriate. 
In conclusion, treatment duration of 14 days may show better results in high metronidazole resistant areas although the optimal treatment duration of bismuth-containing quadruple therapy as second-line is controversial. If moxifloxacin-based triple therapy is decided as second-line therapy, the treatment duration should be 14 days considering high fluoroquinoloneresistance in Korea. According to the European $H$. pylori study group, treatment regimens should achieve the eradication rate over $80 \%$ on ITT basis. ${ }^{46}$ Many capable alternative therapeutic options have been studied, but the choice of an appropriate second-line therapy is a yet challenging task for clinicians. Although 7-BMT, 14-BMT, and 14-MA showed suboptimal efficacies in our study, it may be reasonable to use them as secondline eradication therapy for the Korean population considering high antibiotic resistance. Since the eradication rate of bismuthcontaining quadruple therapy has been decreasing as time progressed and a similar result is expected in moxifloxacin-based triple therapy, further studies are needed to overcome this.

\section{CONFLICTS OF INTEREST}

No potential conflict of interest relevant to this article was reported.

\section{REFERENCES}

1. McColl KE. Clinical practice: Helicobacter pylori infection. N Engl J Med 2010;362:1597-1604.

2. Malfertheiner P, Megraud F, O'Morain C, et al. Current concepts in the management of Helicobacter pylori infection: the Maastricht III Consensus Report. Gut 2007;56:772-781.

3. Chey WD, Wong BC; Practice Parameters Committee of the American College of Gastroenterology. American College of Gastroenterology guideline on the management of Helicobacter pylori infection. Am J Gastroenterol 2007;102:1808-1825.

4. Fischbach W, Malfertheiner P, Hoffmann JC, et al. S3-guideline "Helicobacter pylori and gastroduodenal ulcer disease" of the German Society for Digestive and Metabolic Diseases (DGVS) in cooperation with the German Society for Hygiene and Microbiology, Society for Pediatric Gastroenterology and Nutrition e. V., German Society for Rheumatology, AWMF-registration-no. 021 / 001. Z Gastroenterol 2009;47:1230-1263.

5. Kim BG, Lee DH, Ye BD, et al. Comparison of 7-day and 14-day proton pump inhibitor-containing triple therapy for Helicobacter pylori eradication: neither treatment duration provides acceptable eradication rate in Korea. Helicobacter 2007;12:31-35.

6. Kim N, Kim JJ, Choe YH, et al. Diagnosis and treatment guidelines for Helicobacter pylori infection in Korea. Korean J Gastroenterol 2009;54:269-278.

7. Graham DY, Fischbach L. Helicobacter pylori treatment in the era of increasing antibiotic resistance. Gut 2010;59:1143-1153.

8. Gisbert JP, Pajares R, Pajares JM. Evolution of Helicobacter pylori therapy from a meta-analytical perspective. Helicobacter 2007;12 Suppl 2:50-58.

9. Della Monica P, Lavagna A, Masoero G, Lombardo L, Crocellá L, Pera A. Effectiveness of Helicobacter pylori eradication treatments in a primary care setting in Italy. Aliment Pharmacol Ther 2002;16:1269-1275.

10. O'Connor A, Gisbert JP, McNamara D, O'Morain C. Treatment of Helicobacter pylori infection 2010. Helicobacter 2010;15 Suppl $1: 46-52$.

11. O'Connor A, Gisbert JP, McNamara D, O'Morain C. Treatment of Helicobacter pylori infection 2011. Helicobacter 2011;16 Suppl 1:53-58.

12. Gisbert JP, Calvet X. Review article: the effectiveness of standard triple therapy for Helicobacter pylori has not changed over the last decade, but it is not good enough. Aliment Pharmacol Ther 2011;34:1255-1268.

13. Malfertheiner P, Mégraud F, O'Morain C, et al. Current concepts in the management of Helicobacter pylori infection: the Maastricht 2-2000 Consensus Report. Aliment Pharmacol Ther 2002;16:167180.

14. Hojo M, Miwa H, Nagahara A, Sato N. Pooled analysis on the efficacy of the second-line treatment regimens for Helicobacter pylori infection. Scand J Gastroenterol 2001;36:690-700.

15. Gisbert JP, Pajares JM. Review article: Helicobacter pylori "rescue" regimen when proton pump inhibitor-based triple therapies fail. Aliment Pharmacol Ther 2002;16:1047-1057.

16. Keating GM, Scott LJ. Moxifloxacin: a review of its use in the management of bacterial infections. Drugs 2004;64:2347-2377.

17. Krasemann C, Meyer J, Tillotson G. Evaluation of the clinical microbiology profile of moxifloxacin. Clin Infect Dis 2001;32 Suppl 1:S51-S63.

18. Di Caro S, Ojetti V, Zocco MA, et al. Mono, dual and triple moxifloxacin-based therapies for Helicobacter pylori eradication. Aliment Pharmacol Ther 2002;16:527-532.

19. Nista EC, Candelli M, Zocco MA, et al. Moxifloxacin-based strategies for first-line treatment of Helicobacter pylori infection. Aliment Pharmacol Ther 2005;21:1241-1247.

20. Bago P, Vcev A, Tomic M, Rozankovic M, Marusić M, Bago J. High eradication rate of $\mathrm{H}$. pylori with moxifloxacin-based treatment: a randomized controlled trial. Wien Klin Wochenschr 2007;119:372-378.

21. Cheon JH, Kim N, Lee DH, et al. Efficacy of moxifloxacin-based triple therapy as second-line treatment for Helicobacterpylori infection. Helicobacter 2006;11:46-51.

22. Bago J, Pevec B, Tomić M, Marusić M, Bakula V, Bago P. Secondline treatment for Helicobacter pylori infection based on moxifloxacin triple therapy: a randomized controlled trial. Wien Klin Wochenschr 2009;121:47-52.

23. Li Y, Huang X, Yao L, Shi R, Zhang G. Advantages of moxifloxacin and levofloxacin-based triple therapy for second-line treatments of persistent Helicobacter pylori infection: a meta analysis. Wien Klin Wochenschr 2010;122:413-422. 
24. Kang JM, Kim N, Lee DH, et al. Second-line treatment for Helicobacter pylori infection: 10-day moxifloxacin-based triple therapy versus 2-week quadruple therapy. Helicobacter 2007;12:623-628.

25. Cellini L, Grande R, Di Campli E, Di Bartolomeo S, Capodicasa S, Marzio L. Analysis of genetic variability, antimicrobial susceptibility and virulence markers in Helicobacter pylori identified in Central Italy. Scand J Gastroenterol 2006;41:280-287.

26. Wang LH, Cheng H, Hu FL, Li J. Distribution of gyrA mutations in fluoroquinolone-resistant Helicobacter pylori strains. World J Gastroenterol 2010;16:2272-2277.

27. Chung KH, Lee DH, Jin E, et al. The efficacy of moxifloxacincontaining triple therapy after standard triple, sequential, or concomitant therapy failure for Helicobacter pylori eradication in Korea. Gut Liver 2014;8:605-611.

28. Moon JY, Kim GH, You HS, et al. Levofloxacin, metronidazole, and lansoprazole triple therapy compared to quadruple therapy as a second-line treatment of Helicobacter pylori infection in Korea. Gut Liver 2013;7:406-410.

29. Lee JH, Shin JH, Roe IH, et al. Impact of clarithromycin resistance on eradication of Helicobacter pylori in infected adults. Antimicrob Agents Chemother 2005;49:1600-1603.

30. Kim JY, Kim N, Park HK, et al. Primary antibiotic resistance of Helicobacter pylori strains and eradication rate according to gastroduodenal disease in Korea. Korean J Gastroenterol 2011;58:7481.

31. Mock T, Yatscoff R, Foster R, et al. Clinical validation of the Helikit: a 13C urea breath test used for the diagnosis of Helicobacter pylori infection. Clin Biochem 1999;32:59-63.

32. Megraud F. Helicobacter pylori and antibiotic resistance. Gut 2007;56:1502.

33. Parente F, Cucino C, Bianchi Porro G. Treatment options for patients with Helicobacter pylori infection resistant to one or more eradication attempts. Dig Liver Dis 2003;35:523-528.

34. Mégraud F. H pylori antibiotic resistance: prevalence, importance, and advances in testing. Gut 2004;53:1374-1384.

35. Lee JW, Kim N, Kim JM, et al. Prevalence of primary and second- ary antimicrobial resistance of Helicobacter pylori in Korea from 2003 through 2012. Helicobacter 2013;18:206-214.

36. Mégraud F, Lamouliatte H. Review article: the treatment of refractory Helicobacter pylori infection. Aliment Pharmacol Ther 2003;17:1333-1343.

37. Mégraud F, Lehours P. Helicobacter pylori detection and antimicrobial susceptibility testing. Clin Microbiol Rev 2007;20:280-322.

38. Lee BH, Kim N, Hwang TJ, et al. Bismuth-containing quadruple therapy as second-line treatment for Helicobacter pylori infection: effect of treatment duration and antibiotic resistance on the eradication rate in Korea. Helicobacter 2010;15:38-45.

39. Nishizawa T, Suzuki H, Hibi T. Quinolone-based third-line therapy for Helicobacter pylori eradication. J Clin Biochem Nutr 2009;44:119-124.

40. Yoon H, Kim N, Lee BH, et al. Moxifloxacin-containing triple therapy as second-line treatment for Helicobacter pylori infection: effect of treatment duration and antibiotic resistance on the eradication rate. Helicobacter 2009;14:77-85.

41. Miyachi H, Miki I, Aoyama N, et al. Primary levofloxacin resistance and gyrA/B mutations among Helicobacter pylori in Japan. Helicobacter 2006;11:243-249.

42. Kim JM, Kim JS, Kim N, Jung HC, Song IS. Distribution of fluoroquinolone MICs in Helicobacter pylori strains from Korean patients. J Antimicrob Chemother 2005;56:965-967.

43. Zou J, Yang ZX, Qin ZM. Laboratory and clinical study of levofloxacin against Helicobacter pylori. Zhonghua Yi Xue Za Zhi 2003;83:1778-1781.

44. Chung JW, Lee GH, Jeong JY, et al. Resistance of Helicobacter pylori strains to antibiotics in Korea with a focus on fluoroquinolone resistance. J Gastroenterol Hepatol 2012;27:493-497.

45. Qasim A, O'Morain CA. Review article: treatment of Helicobacter pylori infection and factors influencing eradication. Aliment Pharmacol Ther 2002;16 Suppl 1:24-30.

46. Graham DY, Lu H, Yamaoka Y. A report card to grade Helicobacter pylori therapy. Helicobacter 2007;12:275-278. 